\title{
Sphenoid Sinusitis Associated with Meningitis, Visual Disturbances and Total Ophthalmoplegia
}

\author{
KATSUHIRO MIZOGUCHI, HIROTO JOJIMA, MASAMITSU TANAKA, \\ MARIKO HOTTA, HIDEKI YANO, TERUO TOYOMASU, \\ HIROSHI SHOJI, MASARO KAJI AND MASAHARU KONDO* \\ Departments of Internal Medicine and Bacteriology*, Kurume University School \\ of Medicine, Kurume, 830 Japan
}

Received for publication November 28, 1988

\begin{abstract}
Summary: The sphenoid sinus is deeply situated in the nasal cavity and infection of this sinus is less common than infections of other sinuses. Accordingly, sphenoid sinus infections are frequently misdiagnosed on initial evaluation. The walls of this sinus are adjacent to the pituitary gland, optic nerve and cavernous sinuses. A case of sphenoid sinusitis associated with meningitis, visual disturbances and total ophthalmoplegia was reported. The patient, a 58 year-old female, complained of visual disturbances and deep pain in both retroorbital regions. On admission, the neurological examination revealed a bilateral choked disc with III rd, IV th, Vth and VI th cranial nerve palsies. The cerebrospinal fluid was clear with a pressure of $280 \mathrm{mmH}_{2} \mathrm{O}$, and contained 93 cells per $\mathrm{mm}^{3}$., consisting mainly of lymphocytes. The sugar content was $55 \mathrm{mg}$ $/ \mathrm{dl}$ and the protein content was $344 \mathrm{mg} / \mathrm{dl}$. CT and MRI showed that the sphenoid sinus was filled with pus, but there was no evidence of bone destruction. Surgical drainage was performed for the sphenoid sinusitis. The CSF findings returned to the normal ranges, but the visual disturbance and total ophthalmoplegia still remained, two months after the drainage. The shenoid sinusitis can easily spread to the pituitary gland, optic nerve and cavernous sinuses, and result in serious irreversible neurologic complications. This case demonstrates that early diagnosis and treatment are important.
\end{abstract}

Key words: sphenoid sinusitis - meningitis - optic nerve - cavernous sinus headache

\section{Introduction}

The sphenoid sinus is deeply situated in the nasal cavity, thus infection of this sinus is less common than other sinus infections. Accordingly, sphenoid sinus infections are frequently misdiagnosed during the initial evaluation. The walls of this sinus are adjacent to the pituitary gland, optic nerve and cavernous sinuses, and an infection can easily spread to these sites (Fig. 1).
A case of sphenoid sinusitis associated with meningitis, visual disturbances and total ophthalmoplegia was observed and this case emphasizes the importance of early diagnosis of this infection.

\section{Case report}

A 58 year-old female was admitted to the hospital because of visual disturbances and deep pain in both retroorbital regions. 

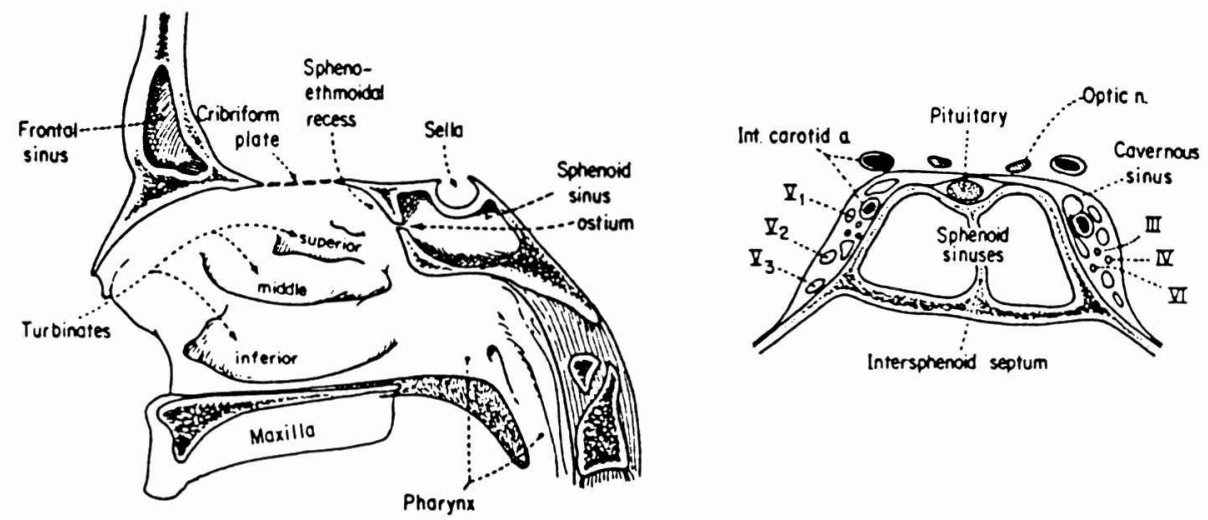

Fig. 1. Anatomical location of the sphenoid sinus. Quotation from Lew, D. et al.

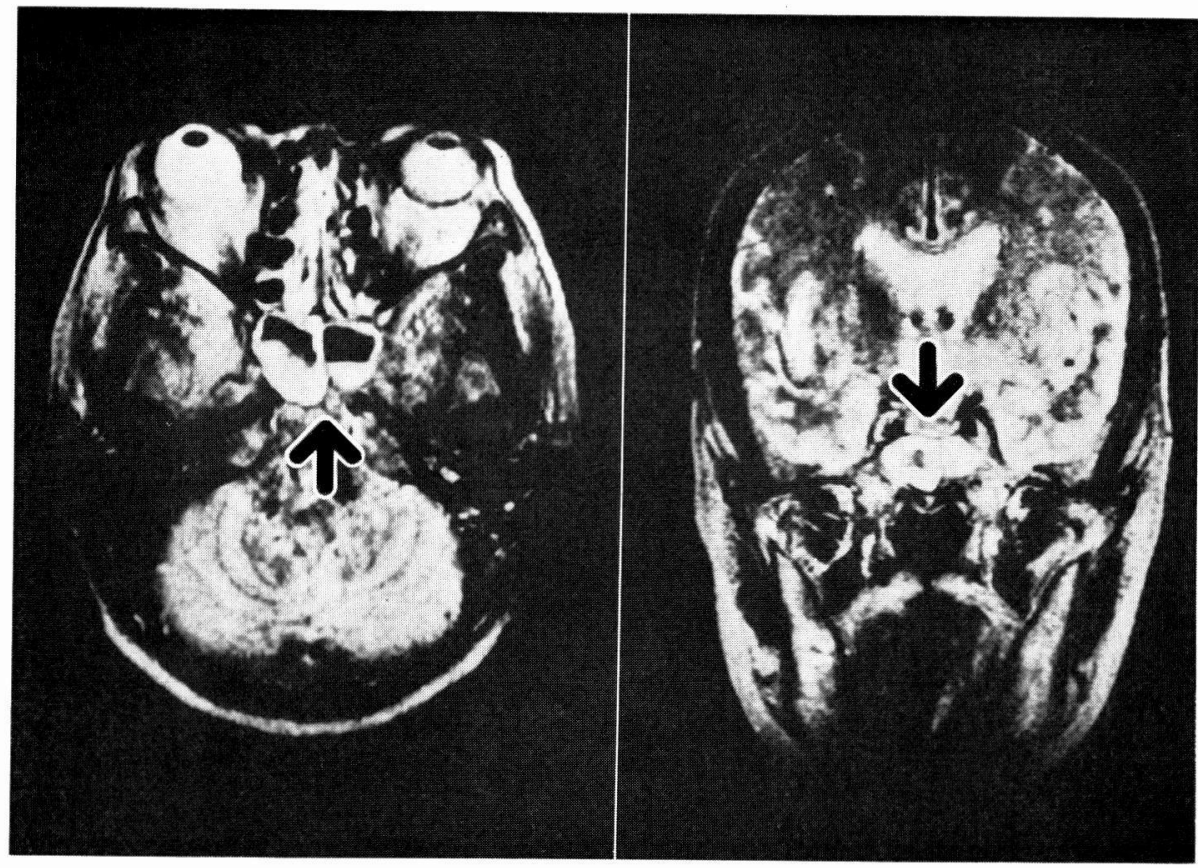

Fig. 2. Magnetic resonance imaging (MRI), showing pus in the bilateral sphenoid sinus, but no bony destruction (arrow).

She had noticed a loss of left visual acuity in May, 1987, and was informed that she had a choked disc. The cerebrospinal fluid pressure was $210 \mathrm{mmH}_{2} \mathrm{O}$, and there were 282 cells per $\mathrm{mm}^{3}$., $80 \%$ of which were lymphocytes. Antibiotics were administered after the initial diagnosis of meningitis was made. The CSF values returned to the normal ranges, and the patient was discharged on Sep. 17, 1987. She was readmitted to the hospital because the deep pain of the retroorbital regions became more severe and the loss of visual acuity progressed. She also experienced nausea 
and vomiting. There was a questionable history of right optic nerve atrophy during childhood and no history of otorhinolaryngological disease.

On admission, the blood pressure was $130 / 70 \mathrm{mmHg}$, the pulse was 78 , and the temperature was $37.2^{\circ} \mathrm{C}$. She had bilateral exophthalmos and ptosis. The chest and abdomen were normal.

The neurological examination revealed that she had a normal level of consiousness, a bilateral loss of visual acuity, exophthalmos, a choked disc, bilateral ptosis and mydriasis $(7 \mathrm{~mm})$, no reflex reaction to light, total ophthalmoplegia and a sensory disturbance in the first division of the Vth nerve. Neck stiffness was negative. The motor systems, sensory systems, reflexes, urination and defecation were normal.

The urine was normal. The hematocrit was 30.0 percent; the white-cell count was 9900. The platelet count was 565,000 , and the erythrocyte sedimentation rate was 131 $\mathrm{mm}$ per hour. Liver and renal function, electrocytes, thyroid function and angiotensin converting enzyme were within normal limits. The blood culture and a screening test for malignancy were negative. X-ray films of the chest, abdomen and skull disclosed no abnormality. A computed tomographic (CT) scan showed that there was almost no air in the bilateral sinuses. The magnetic resonance imaging (MRI) revealed that the bilateral sphenoid sinuses were filled with pus, but there was no evidence of bone destruction (Fig. 2). The cerebrospinal fluid was watery clear and the csf pressure was 280 $\mathrm{mmH}_{2} \mathrm{O}$. The CSF contained 93 cells per $\mathrm{mm}^{3}$., $80 \%$ of which were lymphocytes. The sugar content was $55 \mathrm{mg} / \mathrm{dl}$ and the protein content was $344 \mathrm{mg} / \mathrm{dl}$. Cultures of the cerebrospinal fluid for bacillus, tubercle bacillus and fungus were all negative, and no abnormal cells were found.

\section{Clinical Course}

This case was diagnosed as sphenoid sinusitis associated with aseptic meningitis, visual disturbances and total ophthalmoplegia with general neurological and laboratory findings. On the $43 \mathrm{rd}$ hospital day, the sphenoid sinus was drained surgically because the symptoms and neurological findings persisted in spite of antibiotic treatment. Lactobacillus plantarum and Candida glabrata were isolated from the pus (Fig. 3). Histological examination of the tissue that was removed from the sinus showed only inflammation and never indicated malignancy. The cerebrospinal fluid findings on the 49 th day after surgical drainage returned to the normal
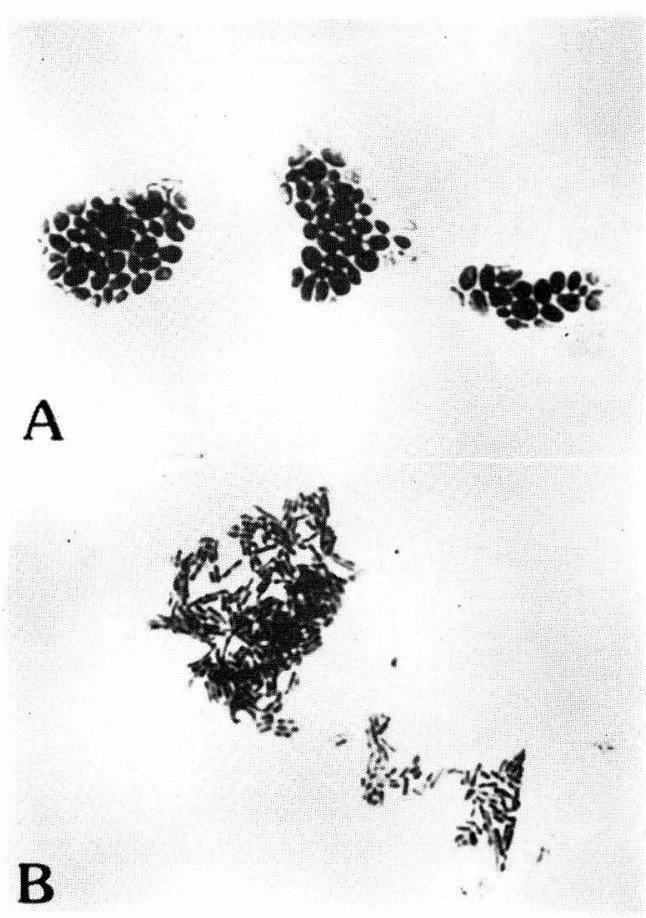

Fig. 3. Culture of pus from the sphenoid sinus. Lactobacillus plantarum (A) and Candida glabrata (B) were present (Gram stain, $\times 400)$. 
ranges, with 3 cells per $\mathrm{mm}^{3}$, a sugar content of $81 \mathrm{mg} / \mathrm{dl}$ and protein of $46 \mathrm{mg}$ /dl. Eye movements were slightly improved, but visual acuity never improved.

\section{Discussion}

This patient had a sphenoid sinusitis associated with aseptic meningitis, visual disturbances and total ophthalmoplegia. The sphenoid sinus is deeply situated in the nasal cavity and an infection at this site is often not observed during a routine radiologic examination. Sphenoid sinusitis is also overlooked because the frequency is low. Lew et al. (1983) reported that sphenoid sinusitis represented only $2.7 \%$ of the total number of sinusitis cases. There is also a case of sphenoid sinusitis where pus was found in the sphenoid sinus by a surgical procedure because the infection was suspected by clinical findings, however there were no indications of an infection on the routine radiologic examinations (Wyllie et al. 1973).

Retroorbital and frontal pain are common initial symptoms that interfere with sleep and are not relieved by aspirin. Thus sphenoid sinusitis is often misdiagnosed as atypical facial pain, migraine headache or trigeminal neuralgia. Lew et al. (1983) obtained cerebrospinal fluid from ten of thirty patients with sphenoid sinusitis. In five, the findings were typical of a parameningeal infection. Findings in the other five patients were indicative of bacterial meningitis. Cultures of cerebrospinal fluid from four patients were positive. In each case, the same pathogen was found in the sphenoid sinus and the cerebrospinal fluid. Cultures of the cerebrospinal fluid from our patients were negative for bacilli. Only the culture of the pus in the sphenoid sinus was positive. Teed (1938) reported that meningitis was the predominant complication in 173 patients with sphenoid sinusitis. As the sphenoid sinusitis slowly progresses, a proliferative osteomyelitis forms around the sphenoid cavity. With time the natural ostium becomes completely occluded and the pressure increases, bone proliferation can account for the prolonged and severe symptoms, because the walls of this sinus are adjacent to the pituitary gland, optic nerve and the cavernous sinuses (Cody CC III, 1956). The fact that the sphenoid walls are thin and occasionally the bone is defective make it possible for a lesion in the sphenoid sinus to easily spread to adjacent structures. In this case, the sphenoid sinusitis progressed, chronically, and the infection spread to the cavernous sinuses. Though angiography was not performed, the possibility of sphenoid sinusitis must be considered because of the visual disturbances and the total ophthalmoplegia. Also a case has been reported (Nelson et al. 1967) in which an infection had spread to the pituitary gland and the patient died of pituitary dysfunction. Candida glabrata and Lactobacillus plantarum, both obligate anaerobes, were isolated from the pus in the sphenoid sinus in the present case. Lactobacillus plantarum meningitis has not been reported; but one' case of Candida glabrata meningitis has been reported in a patient who had diabetes mellitus and required prolonged treatment with antibiotics (Anhalt et al. 1986). In this case, we suspected an infection in the sphenoid sinus in spite of the antibiotic treatment. As the natural ostium become occluded, the air in the sphenoid sinus was removed and that made it possible for the Candida glabrata and Lactobacillus plantarum, which are anaerobic, to proliferate and finally become absessed. According to Lew et al. (1983), four of fifteen patients with acute sphenoid sinusitis did not undergo surgery for drainage of the sinus. These patients died of bacterial meningitis and cavernous sinus thrombosis. With one exeption, sphenoid sinusitis was not suspected while they were alive. 
Sphenoid sinusitis is frequently overlooked, as described above, and exists though the maxillary sinus is normal, as in this case. If a patient has retroorbital pain that can not be relieved by aspirin and diplopia, sphenoid sinusitis must be suspected. Early diagnosis and aggresive therapy, including surgical drainage, is critical for prevention of the frequently fatal complications of this infection.

\section{References}

Anhalt, E., Alvarez, J. and Berg, R. (1986).
Candida glabrata meningitis. South Med. J. 79, 916.

Cody, C. C. III. (1956). An unusual case of sphenoid abscess. Arch. Otolaryngol. 63, 199-202.

Lew, D., Southwick, F.S., Montgomery, W.W., Weber, A. L. and Baker, A.S. (1983). Sphenoid sinusitis. N. Engl. J. Med. 309, 1149-1154.

Nelson, D. A., Holloway, W. J., Kara-Eneff, S.C. and GoldenBerg, H. I. (1967). Neurological syndromes produced by sphenoid sinus abscess. Neurology 17, 981-987.

TEED, R.W. (1938). Meningitis from the sphenoid sinus. Arch. Otolaryngol. 28, 589-617.

Wyllie, J. W. III., Kern, E. B. and DJalilian, M. (1973). Isolated sphenoid sinus lesions. Laryngoscope 83, 1252-1265. 